\title{
A geometric integral formulation for eddy-currents
}

\author{
L. Codecasa ${ }^{1}$, R. Specogna ${ }^{2}$ and F. Trevisan ${ }^{2, *, \dagger}$ \\ ${ }^{1}$ Politecnico di Milano, Piazza Leonardo da Vinci 32, I-20133 Milan, Italy \\ ${ }^{2}$ Università di Udine, Via delle Scienze 208, I-33100 Udine, Italy
}

\begin{abstract}
SUMMARY
In the computational electromagnetism community it is known how the differential formulation of an eddy-current problem, can be translated into a finite dimensional system of equations involving circulations and fluxes, by means of the so-called Discrete Geometric Approach. This is done by exploiting the geometric structure behind Maxwell's equations. In this paper, we will show how the same Discrete Geometric Approach can be profitably used also to discretize an eddy-current problem formulated in an integral way.

We rely on a purely geometric definition of a novel set of face vector basis functions that we use to construct the discrete counterparts-matrices - of both the Ohm's constitutive relation and of the integral relation between the magnetic vector potential and the eddy-current density vector. The symmetry and positive-definiteness of such matrices will be demonstrated and their geometric structure will be apparent. Copyright (c) 2010 John Wiley \& Sons, Ltd.
\end{abstract}

Received 29 October 2008; Revised 31 August 2009; Accepted 25 November 2009

KEY WORDS: cell method; integral formulations; eddy-currents

\section{INTRODUCTION}

The discretization of a magnetoquasistatic problem formulated in terms of partial differential equations is commonly and conveniently performed by means of Finite Element techniques. More recently, the so-called Discrete Geometric Approaches put the emphasis on the geometric structure behind Maxwell's equations and they made visible how the basic laws of eddy-currents-and in general of electromagnetism - can be stated directly in an algebraic form, in terms of circulations and fluxes of the related field quantities, plus the discrete counterparts of the constitutive relations. (For a background of the discrete geometric approach, see papers by Weiland, Tonti and Bossavit, such as [1-10] and also papers such as [11-13].) With a particular choice of the base functionsthe Whitney vector base functions-this modern Discrete Geometric Approach allows to reobtain

\footnotetext{
*Correspondence to: F. Trevisan, Università di Udine, Via delle Scienze 208, I-33100 Udine, Italy.

†E-mail: trevisan@uniud.it

Contract/grant sponsor: Publishing Arts Research Council; contract/grant number: 98-1846389
}

Copyright (C) 2010 John Wiley \& Sons, Ltd. 
a finite dimensional system of equations of the same kind as that given by the classical Galerkin approach in Finite Elements [14, 15].

On the other hand, a magnetoquasistatic problem can be also formulated in terms of integral equations [16-18]. Integral equations allow, in general, to concentrate the mesh in the conducting and magnetic regions only and regularity conditions of the field at the infinity are automatically satisfied; as a drawback, they lead to a full system of equations, compared with the sparse system yielded by the discretization of partial differential equations.

Without entering in the competition between the two ways of formulating an electromagnetic eddy-current problem, the aim of this paper is to show how a discrete geometric formulation is possible also for an integrally formulated magnetoquasistatic problem. We will concentrate mainly on the geometric aspects at the base of the integral formulation and on the discretization process which yields to a discrete differential system of equations from the original integral equation governing an eddy-current problem in linear media. The resulting integral geometric formulation can also treat non-topologically trivial domains by resorting to the techniques already described originally in $[16,19]$ for multiply connected regions or more generally in [20-22] and we will not give the details here.

We will start with the continuity law, Ampère's law and Faraday's law stated in discrete setting; then the matrices representing the discrete counterparts of Ohm's constitutive relation and of the integral relation between the magnetic vector potential and current density vector will be computed by introducing a novel fully geometric set of face vector functions defined not only for tetrahedralike Whitney face vector functions are-but also for (oblique) prisms with triangular base and (oblique) parallelepipeds. We will show how the elements of these matrices are directly related to the edge vectors associated with the edges of the mesh.

The paper is organized as follows. Section 2 describes the geometric integral formulation for eddy-current and the final linear differential system of equations. In Section 3 the novel face vector functions for tetrahedra, (oblique) triangular prisms and (oblique) parallelepipeds are described in a geometric way. In Sections 4 and 5 the discrete counterpart of Ohm's law and of the integral relation between the magnetic vector potential and the current density vector are constructed, respectively. The numerical solution of a 3D eddy-current problem compared with a reference finite elements solution will conclude the paper.

\section{THE GEOMETRIC INTEGRAL FORMULATION}

We consider an eddy-current problem in a domain $\Omega$, where a conducting region $\Omega_{\mathrm{c}}$ is present with resistivity $\eta(\boldsymbol{r})$, function of position vector $\boldsymbol{r}$, together with a source region $\Omega_{\mathrm{s}}$. The insulating region is $\Omega_{\mathrm{a}}=\Omega-\Omega_{\mathrm{c}} \cup \Omega_{\mathrm{s}}$. The magnetic medium in $\Omega$ is assumed linear with uniform permeability $\mu$.

The spatial region $\Omega_{\mathrm{c}}$ is covered by a pair of oriented grids $\mathscr{G}, \tilde{\mathscr{G}}[4,14]$. The grid $\mathscr{G}$ consists of tetrahedra, (oblique) triangular prisms and (oblique) parallelepipeds; it has $n$ nodes, $l$ edges, $f$ faces and $v$ volumes. Each of these geometrical elements is given an orientation. The other grid $\tilde{\mathscr{G}}$ is constructed according to the barycentric subdivision of $\mathscr{G}$ [23]; it has $\tilde{n}=v$ nodes, $\tilde{l}=f$ edges, $\tilde{f}=l$ faces and $\tilde{v}=n$ volumes. The orientation of each geometrical element of $\tilde{\mathscr{G}}$ is induced by the orientation of the corresponding geometrical element of the grid $\mathscr{G}$. This one-to-one correspondence between the geometrical elements of $\mathscr{G}, \tilde{G}$ is often referred to as duality. 
The electromagnetic field quantities of the eddy-current problem are discretized into integral quantities associated with the geometrical elements of the pair of staggered grids $\mathscr{G}, \tilde{\mathscr{G}}$; the integral quantities associated with the geometrical elements of $\tilde{\mathscr{G}}$ will be marked with a tilde above. We introduce the $f \times 1$ array $\mathbf{i}(t)$ whose elements are the currents $i_{i}(t)$, with $i=1 \ldots f$, sampled through the $f$ faces of $\mathscr{G}$; such an array is dependent from the grain $h$ of the grid $\mathscr{G}$ and it will be involved in the construction of the finite-dimensional system of equations. Conceptually, the array $\mathbf{i}(t)$ only approximates (in the limit for $h \rightarrow 0$, it converges to) the actual array $\rho_{f} \boldsymbol{j}(\boldsymbol{r}, t)$ of dimension $f \times 1$ of the fluxes of the current density $\boldsymbol{j}(\boldsymbol{r}, t)$ in $\Omega_{\mathrm{c}}$ through the $f$ faces of $\mathscr{G}$, where symbol $\rho_{f}$ acts on a vector field $(\boldsymbol{j}(\boldsymbol{r}, t)$ in the specific case) yielding an array of fluxes (currents in the specific case) through the faces of $\mathscr{G}$.

The integral quantities associated with the geometrical elements of $\mathscr{G}, \tilde{\mathscr{G}}$ are related by balance equations which translate in an algebraic form the physical laws ruling an eddy-current problem. Therefore, we begin with continuity law which involves the array $\mathbf{i}(t)$; it is discretized into the following matrix equation:

$$
\operatorname{Di}(t)=0,
$$

where matrix $\mathbf{D}$ denotes the $v \times f$ volume-face incidence matrix of the grid $\mathscr{G}$.

The discrete counterpart of the boundary condition $\boldsymbol{j}(\boldsymbol{r}, t) \cdot \boldsymbol{n}=0$ on $\partial \Omega_{\mathrm{c}}$ can be written as $i_{i}(t)=0$ with $i=1, \ldots, f_{b}$, where $f_{b}$ is the number of faces of $\mathscr{G}$ on $\partial \Omega_{\mathrm{c}}$ and we can introduce a reduced array $\mathbf{i}_{\mathrm{r}}(t)$ of dimension $\left(f-f_{b}\right) \times 1$ obtained from $\mathbf{i}(t)$ by eliminating the rows associated with the faces of $\mathscr{G}$ on $\partial \Omega_{\mathrm{c}}$.

To satisfy (1), we search for an $l \times 1$ array $\mathbf{T}(t)$, of elements $T_{i}(t)$ with $i=1 \ldots l$, which approximates the $l \times 1$ array $\rho_{e} \boldsymbol{T}(\boldsymbol{r}, t)$ of the circulations of the electric vector potential $\boldsymbol{T}(\boldsymbol{r}, t)$ along the edges of $\mathscr{G}$ such that Ampère's law can be written as

$$
\mathbf{i}(t)=\mathbf{C T}(t),
$$

where $\mathbf{C}$ is the $f \times l$ face-edge incidence matrix for the grid $\mathscr{G}$. The discrete counterpart of the boundary condition $i_{i}(t)=0$ with $i=1, \ldots, f_{b}$ for the $f_{b}$ faces of $\mathscr{G}_{\text {on }} \partial \Omega_{\mathrm{c}}$, translates into a number of linear equations involving the $T_{i}(t)$, with $i=1, \ldots, l_{b}$, associated with the $l_{b}$ edges forming $\partial \Omega_{c}$; for a topologically trivial domain $\Omega_{\mathrm{c}}$, we write $l_{b}$ equations $T_{i}(t)=0$, with $i=1, \ldots, l_{b}$. Otherwise for a non-trivial domain, we resort to the classical technique based on the cuts computation, for the details refer to [20-23]. According to this technique an additional unknown $T_{\mathrm{c}}$ is associated with each cut $c$, with $c=1, \ldots, C, C$ being the number of cuts; correspondingly an additional equation is added per each cut $c$, often referred to as non-local equation [22]. As a consequence, all the $T_{i}$ values, with $i=1, \ldots, l_{b}$, associated with the $l_{b}$ edges on $\partial \Omega_{p}$, have a prescribed value ${ }^{\S}$ and they can be eliminated from the array $\mathbf{T}(t)$ yielding a reduced array $\mathbf{T}_{\mathbf{r}}(t)$ of dimension $\left(l-l_{b}\right) \times 1$.

Now we can rewrite (2) in terms of the reduced arrays

$$
\mathbf{i}_{\mathrm{r}}(t)=\mathbf{C}_{\mathrm{r}} \mathbf{T}_{\mathrm{r}}(t),
$$

\footnotetext{
${ }^{\ddagger}$ Symbol $\rho_{e}$ acts on a vector field yielding an array of circulations along the edges of $\mathscr{G}$.

${ }^{\S}$ This value is null in a trivial domain. For a non-trivial domain this value is non-null only for the $T_{i}$ associated with the edges of the so-called ribbon corresponding to cut $c$; in this case $T_{i}= \pm T_{\mathrm{c}}$ holds, where $T_{\mathrm{c}}$ is the additional unknown for the cut $c$ and +1 holds when the orientations of the ribbon edge $e_{i}$ and of the reference edge $e_{\mathrm{c}}$ match.
} 
where $\mathbf{C}_{\mathrm{r}}$ is the reduced incidence matrix of dimension $\left(f-f_{b}\right) \times\left(l-l_{b}\right)$ obtained from $\mathbf{C}$ by eliminating the $f_{b}$ rows and $l_{b}$ columns associated with the faces and edges of $\mathscr{G}$ on $\partial \Omega_{\mathrm{c}}$, respectively.

Thanks to the linearity and homogeneity of the magnetic medium, we can introduce a magnetic vector potential $\boldsymbol{A}(\boldsymbol{r}, t)$ due to $\boldsymbol{j}(\boldsymbol{r}, t)$ in $\Omega_{\mathrm{c}}$ as

$$
\boldsymbol{A}(\boldsymbol{r}, t)=\frac{\mu}{4 \pi} \int_{\Omega_{\mathrm{c}}} \frac{\boldsymbol{j}(\boldsymbol{p}, t)}{|\boldsymbol{r}-\boldsymbol{p}|} \mathrm{d} \Omega_{\boldsymbol{p}}
$$

and the vector potential $\boldsymbol{A}_{\mathrm{s}}(\boldsymbol{r}, t)$ due to the known source current density $\boldsymbol{j}_{\mathrm{s}}(\boldsymbol{r}, t)$ in $\Omega_{\mathrm{s}}$ as

$$
\boldsymbol{A}_{\mathrm{S}}(\boldsymbol{r}, t)=\frac{\mu}{4 \pi} \int_{\Omega_{\mathrm{s}}} \frac{\boldsymbol{j}_{\mathrm{s}}(\boldsymbol{p}, t)}{|\boldsymbol{r}-\boldsymbol{p}|} \mathrm{d} \Omega_{\boldsymbol{p}}
$$

where we denoted with $d \Omega_{p}$ the volume differential around $\boldsymbol{p}$. As it is well known, the total vector potential $\boldsymbol{A}(\boldsymbol{r}, t)+\boldsymbol{A}_{\mathrm{S}}(\boldsymbol{r}, t)$ is a solution of the canonical magnetostatic problem in $\Omega$ with solenoidal sources $\boldsymbol{j}(\boldsymbol{r}, t)$ and $\boldsymbol{j}_{\mathrm{s}}(\boldsymbol{r}, t)$ in $\Omega_{\mathrm{c}}$ and $\Omega_{\mathrm{s}}$, respectively.

We introduce now the $\tilde{f} \times 1$ array $\tilde{\varphi}(t)$, of elements $\tilde{\varphi}_{i}(t)$ with $i=1, \ldots, \tilde{f}$, which approximates the $\tilde{f} \times 1$ array ${ }^{\mathbb{l}} \rho_{\tilde{f}} \boldsymbol{b}(\boldsymbol{r}, t)$ of the fluxes of the magnetic induction $\boldsymbol{b}(\boldsymbol{r}, t)$ through the faces of

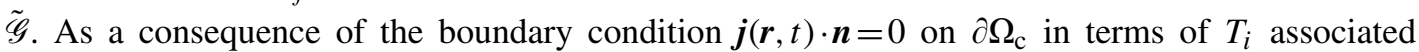
with the edges $l_{i}$ of $\mathscr{G}$ on $\partial \Omega_{\mathrm{c}}$, with $i=1, \ldots, l_{b}$, we may consider the reduced array $\tilde{\varphi}_{\mathrm{r}}(t)$ of dimension $\left(l-l_{b}\right) \times 1$ obtained from $\tilde{\varphi}(t)$ by eliminating the $l_{b}$ fluxes on the faces of $\tilde{\mathscr{G}}$ in a one-to-one correspondence with the $l_{b}$ edges of $\mathscr{G}$ on $\partial \Omega_{\mathrm{c}}$. The $\tilde{l} \times 1$ array $\tilde{\mathbf{v}}(t)$, of elements $\tilde{v}_{i}(t)$ with $i=1, \ldots, \tilde{l}$, which approximates the $\tilde{l} \times 1$ array ${ }^{\|} \rho_{\tilde{e}} \boldsymbol{e}(\boldsymbol{r}, t)$ of the circulations of the electric field $\boldsymbol{e}(\boldsymbol{r}, t)$ along the edges of $\tilde{\mathscr{G}}$ is also introduced together with the corresponding reduced array $\tilde{\mathbf{v}}_{\mathrm{r}}(t)$ of dimension $\left(f-f_{b}\right) \times 1$ obtained from $\tilde{\mathbf{v}}(t)$ by eliminating the $f_{b}$ voltages associated with edges of $\tilde{\mathscr{G}}$ in a one-to-one correspondence with the $f_{b}$ faces of $\mathscr{G}$ on $\partial \Omega_{\mathrm{c}}$.

Now, Faraday's law is written only for those faces of $\mathscr{G}$ in a one-to-one correspondence with an edge of $\mathscr{G}$ internal to $\Omega_{\mathrm{c}}$; we obtain the following matrix equation:

$$
\mathbf{C}_{\mathrm{r}}^{\mathrm{T}} \tilde{\mathbf{v}}_{\mathrm{r}}(t)=-\frac{d \tilde{\boldsymbol{\varphi}}_{\mathrm{r}}(t)}{\mathrm{d} t}
$$

Next, we introduce the $\tilde{l} \times 1$ array $\tilde{\mathbf{a}}(t)$, of elements $\tilde{a}_{i}(t)$ with $i=1, \ldots, \tilde{l}$, which approximates the $\tilde{l} \times 1$ array $\rho_{\tilde{e}} \boldsymbol{A}(\boldsymbol{r}, t)$ of the circulations of the magnetic vector potential $\boldsymbol{A}(\boldsymbol{r}, t)$ along the edges of $\tilde{\mathscr{G}}$; the array $\rho_{\tilde{e}} \boldsymbol{A}_{\mathrm{s}}(\boldsymbol{r}, t)$ of the circulations of the magnetic vector potential $\boldsymbol{A}_{\mathrm{s}}(\boldsymbol{r}, t)$ along the edges of $\tilde{G}$ will be regarded as a known term.

By eliminating from the array $\tilde{\mathbf{a}}(t)$ the $f_{b}$ circulations associated with edges of $\tilde{\mathscr{G}}$ in a one-to-one correspondence with the $f_{b}$ faces of $\mathscr{G}$ on $\partial \Omega_{\mathrm{c}}$, we obtain the corresponding reduced array $\tilde{\mathbf{a}}_{\mathrm{r}}(t)$ of dimension $\left(f-f_{b}\right) \times 1$. We have that

$$
\tilde{\boldsymbol{\varphi}}_{\mathrm{r}}(t)=\mathbf{C}_{\mathrm{r}}^{\mathrm{T}}\left(\tilde{\mathbf{a}}_{\mathrm{r}}(t)+\rho_{\tilde{e}_{\mathrm{r}}} \boldsymbol{A}_{\mathrm{S}}(\boldsymbol{r}, t)\right)
$$

\footnotetext{
${ }^{I_{S}}$ Symbol $\rho_{\tilde{f}}$ acts on a vector field yielding an array of fluxes through the faces of $\tilde{\mathscr{G}}$.

${ }^{\|}$Symbol $\rho_{\tilde{e}}$ acts on a vector field yielding an array of circulations along the edges of $\tilde{\mathscr{G}}$. 
is the discrete counterpart of the representation of the magnetic induction in terms of the vector potential $\boldsymbol{b}(\boldsymbol{r}, t)=\operatorname{rot}\left(\boldsymbol{A}(\boldsymbol{r}, t)+\boldsymbol{A}_{\mathrm{s}}(\boldsymbol{r}, t)\right)$ inside $\Omega_{\mathrm{c}}$, where $\rho_{\tilde{e}_{\mathrm{r}}} A_{\mathrm{s}}(\boldsymbol{r}, t)$ is the reduced array corresponding to $\rho_{\tilde{e}} \boldsymbol{A}_{\mathrm{s}}(\boldsymbol{r}, t)$.

Ohm's constitutive relation $\boldsymbol{e}(\boldsymbol{r}, t)=\boldsymbol{\eta}(\boldsymbol{r}) \boldsymbol{j}(\boldsymbol{r}, t)$ is discretized into the following matrix equation:

$$
\tilde{\mathbf{v}}(t)=\mathbf{E i}(t),
$$

in which the $f \times f$ matrix $\mathbf{E}$ is a discrete counterpart of the resistivity tensor $\boldsymbol{\eta}(\boldsymbol{r})$; correspondingly, in terms of reduced arrays we write

$$
\tilde{\mathbf{v}}_{\mathrm{r}}(t)=\mathbf{E}_{\mathbf{r}} \mathbf{i}_{\mathrm{r}}(t),
$$

where $\mathbf{E}_{\mathrm{r}}$ is the reduced matrix of dimension $\left(f-f_{b}\right) \times\left(f-f_{b}\right)$ obtained from $\mathbf{E}$ by eliminating the $f_{b}$ rows and columns associated with the faces of $\mathscr{G}$ on $\partial \Omega_{\mathrm{c}}$.

Similarly (4) can be discretized into the following matrix equation:

$$
\tilde{\mathbf{a}}(t)=\mathbf{M i}(t),
$$

where $\mathbf{M}$ is a matrix of dimension $f \times f$; in terms of the corresponding reduced arrays it becomes

$$
\tilde{\mathbf{a}}_{\mathrm{r}}(t)=\mathbf{M}_{\mathrm{r}} \mathbf{i}_{\mathrm{r}}(t)
$$

where $\mathbf{M}_{\mathrm{r}}$ is the reduced matrix of dimension $\left(f-f_{b}\right) \times\left(f-f_{b}\right)$ obtained from $\mathbf{M}$ by eliminating the $f_{b}$ rows and columns associated with the faces of $\mathscr{G}$ on $\partial \Omega_{\mathrm{c}}$.

The problem of discretizing such constitutive relations is crucial and will be faced in a fully geometric way in the following sections.

It is here noted that (3), (6) and (7), descend from similar equations, exactly satisfied by $\rho_{f} \boldsymbol{j}(\boldsymbol{r}, t)$, $\rho_{e} \boldsymbol{T}(\boldsymbol{r}, t), \rho_{\tilde{e}} \boldsymbol{e}(\boldsymbol{r}, t), \rho_{\tilde{f}} \boldsymbol{b}(\boldsymbol{r}, t)$ and by $\rho_{\tilde{e}}\left(\boldsymbol{A}(\boldsymbol{r}, t)+\boldsymbol{A}_{\mathrm{s}}(\boldsymbol{r}, t)\right)$, respectively,

$$
\begin{aligned}
\rho_{f} \boldsymbol{j}(\boldsymbol{r}, t) & =\mathbf{C} \rho_{e} \boldsymbol{T}(\boldsymbol{r}, t), \\
\tilde{\mathbf{C}} \rho_{\tilde{e}} \boldsymbol{e}(\boldsymbol{r}, t) & =-\frac{\mathrm{d}}{\mathrm{d} t} \rho_{\tilde{f}} \boldsymbol{b}(\boldsymbol{r}, t), \\
\rho_{\tilde{f}} \boldsymbol{b}(\boldsymbol{r}, t) & =\tilde{\mathbf{C}} \rho_{\tilde{e}}\left(\boldsymbol{A}(\boldsymbol{r}, t)+\boldsymbol{A}_{\mathbf{s}}(\boldsymbol{r}, t)\right) .
\end{aligned}
$$

On the contrary, the equations obtained from (8), (10) by substituting $\rho_{f} \boldsymbol{j}(\boldsymbol{r}, t)$ for $\mathbf{i}(t)$, and, respectively, $\rho_{\tilde{e}} \boldsymbol{e}(\boldsymbol{r}, t)$ for $\tilde{\mathbf{v}}(t)$ and $\rho_{\tilde{e}} \boldsymbol{A}(\boldsymbol{r}, t)$ for $\tilde{\mathbf{a}}(t)$ are only approximate [14]. This is the wellknown constitutive error affecting the overall discrete formulated electromagnetic problem.

By substituting in (6), (9) for $\tilde{\mathbf{v}}_{\mathrm{r}}(t)$, (7) for $\tilde{\boldsymbol{\varphi}}(t),(11)$ and (3) for $\mathbf{i}(t)$, we obtain the final linear differential system

$$
\mathbf{C}_{\mathrm{r}}^{\mathrm{T}} \mathbf{M}_{\mathrm{r}} \mathbf{C}_{\mathrm{r}} \frac{\mathrm{d}}{\mathrm{d} t} \mathbf{T}_{\mathrm{r}}(t)+\mathbf{C}_{\mathrm{r}}^{\mathrm{T}} \mathbf{N}_{\mathrm{r}} \mathbf{C}_{\mathrm{r}} \mathbf{T}_{\mathrm{r}}(t)=-\mathbf{C}_{\mathrm{r}}^{\mathrm{T}} \frac{\mathrm{d}}{\mathrm{d} t} \rho_{\tilde{e}_{\mathrm{r}}} \boldsymbol{A}_{\mathrm{s}}(\boldsymbol{r}, t) .
$$

For a non-trivial domain, additional equations must be added (one for each computed cut) involving an additional unknown for each cut; to close the integral eddy-current formulation, initial conditions are also imposed. 


\section{GEOMETRIC FACE VECTOR FUNCTIONS}

We introduce the restrictions $\mathscr{G}^{k}, \tilde{\mathscr{G}}^{k}$ of the pair of grids $\mathscr{G}, \tilde{\mathscr{G}}$ to the subdomain $\Omega^{k}$ of $\mathscr{G}$, Figure 1 . Let $\Gamma_{i}^{k}, \tilde{\Sigma}_{i}^{k}$ with $i=1, \ldots, l^{k}$ be the $l^{k}$ edges of $\mathscr{G}^{k}$ and the faces of $\tilde{\mathscr{G}}^{k}$, with $k=1, \ldots, v$, respectively. Similarly let $\Sigma_{i}^{k}, \tilde{\Gamma}_{i}^{k}$ with $i=1, \ldots, f^{k}$ be the $f^{k}$ faces of $\mathscr{G}^{k}$ and the edges of $\tilde{\mathscr{G}}^{k}$, with $k=1, \ldots, v$, respectively. Let $\boldsymbol{l}_{i}^{k}, \tilde{\boldsymbol{s}}_{i}^{k}$ with $i=1, \ldots, l^{k}$ be, respectively, the edge vectors of the edges $\Gamma_{i}^{k}$ of $\mathscr{G}^{k}$ and the face vectors of the faces $\tilde{\Sigma}_{i}^{k}$ of $\tilde{\mathscr{G}}^{k}$. Let $\boldsymbol{s}_{i}^{k}, \tilde{\boldsymbol{l}}_{i}^{k}$ with $i=1, \ldots, f^{k}$ be, respectively, the face vectors of the faces $\Sigma_{i}^{k}$ of $\mathscr{G}^{k}$ and the edge vectors of the edges $\tilde{\Gamma}_{i}^{k}$ of $\tilde{\mathscr{G}}^{k}$. Let $\boldsymbol{r}^{k}$ be the node of $\tilde{\mathscr{G}}^{k}$ which coincides with the barycenter of $\mathscr{G}^{k}$, with $k=1, \ldots, v$.

Let $\tilde{\Omega}_{i}^{k}$ be the volumes of $\tilde{\mathscr{G}}^{k}$, with $i=1, \ldots, n^{k}, n^{k}$ being the number of nodes of $\mathscr{G}^{k}$. For each volume $\tilde{\Omega}_{i}^{k}$, a triple of edges is introduced given by the edges of $\mathscr{G}^{k}$ intersecting $\tilde{\Omega}_{i}^{k}$. Such edges are named $\hat{\Gamma}_{\alpha}^{k}$, with $\alpha=1, \ldots, 3 n^{k}$, and are independently oriented with respect to the edges of $\mathscr{G}^{k}$. Similarly for each volume $\tilde{\Omega}_{i}^{k}$ a triple of faces is introduced given by the faces of $\mathscr{G}^{k}$ intersecting $\tilde{\Omega}_{i}^{k}$.

It is assumed that edge $\hat{\Gamma}_{\alpha}^{k}$ and face $\hat{\Sigma}_{\alpha}^{k}$ with the same index $\alpha$ correspond to a same volume $\tilde{\Omega}_{i}^{k}$, and are not coplanar. Besides it is assumed that $\hat{\Gamma}_{\alpha}^{k}, \hat{\Sigma}_{\alpha}^{k}$ are oriented in such a way that $\hat{\boldsymbol{l}}_{\alpha}^{k} \cdot \hat{\boldsymbol{s}}_{\alpha}^{k}>0$, in which $\hat{\boldsymbol{l}}_{\alpha}^{k}$ is the edge vector of $\hat{\Gamma}_{\alpha}^{k}$ and $\hat{\boldsymbol{s}}_{\alpha}^{k}$ is the face vector of $\hat{\Sigma}_{\alpha}^{k}$.

In this way a triplet of edges $\hat{\Gamma}_{\alpha}^{k}$ and a triplet of faces $\hat{\Sigma}_{\alpha}^{k}$ univocally correspond to $\tilde{\Omega}_{i}^{k}$. This correspondence will be described with a function $i=n(\alpha)$ which associates with each pair of an edge $\hat{\Gamma}_{\alpha}^{k}$ and a face $\hat{\Sigma}_{\alpha}^{k}$ the corresponding volume $\tilde{\Omega}_{i}^{k}$.

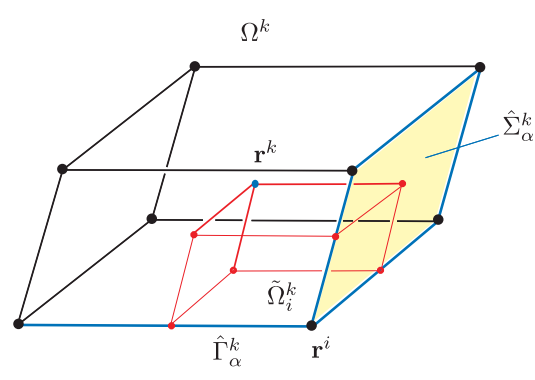

(a)

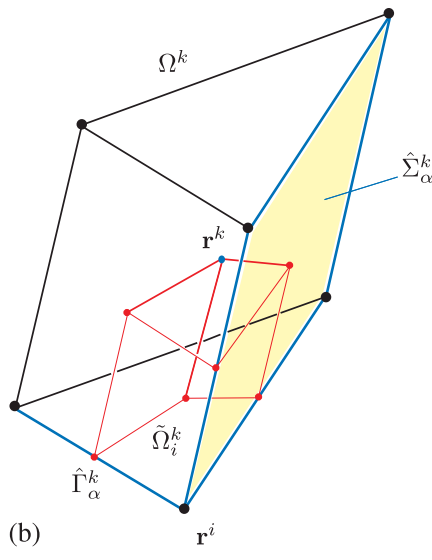

(b)

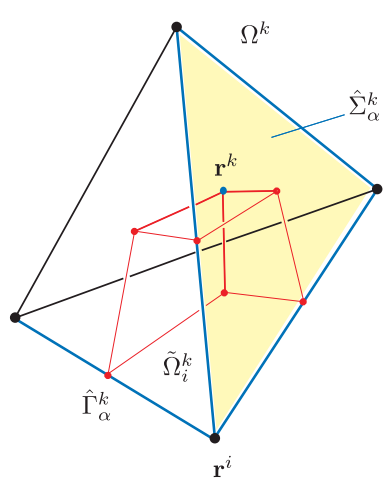

(c)

Figure 1. The three typologies of $\Omega^{k}$ volumes are shown: (oblique) parallelepiped (a), (oblique) triangular prism (b) and tetrahedron (c). The pair of not coplanar edge $\hat{\Gamma}_{\alpha}^{k}$ and face $\hat{\Sigma}_{\alpha}^{k}$ corresponding to the same dual volume $\tilde{\Omega}_{i}^{k}$, with $i=n(\alpha)$ are also evidenced. 
Let $\hat{K}^{k}$ be $\frac{1}{8}, \frac{1}{12}, \frac{1}{24}$ according to the volume $\Omega^{k}$ is an (oblique) parallelepiped, an (oblique) triangular prism or a tetrahedron, respectively. Besides let $\hat{C}_{\alpha}^{k}$ be 1 or 2 according to the face $\hat{\Sigma}_{\alpha}^{k}$ is a parallelogram or a triangle. Let $\hat{\mathbf{P}}^{k}$ be the $3 n^{k} \times f^{k}$ rectangular matrix whose elements are

$$
\hat{p}_{\alpha i}^{k}=\frac{\hat{\boldsymbol{s}}_{\alpha}^{k}}{\left|\hat{\boldsymbol{s}}_{\alpha}^{k}\right|} \cdot \frac{\boldsymbol{s}_{i}^{k}}{\left|\boldsymbol{s}_{i}^{k}\right|} \delta_{f(\alpha) i},
$$

$f(\alpha)$ being the index of the face $\Sigma_{f(\alpha)}^{k}$ of $\mathscr{G}^{k}$ corresponding to face $\hat{\Sigma}_{\alpha}^{k}$ and $i=1, \ldots, f^{k}$.

\section{Lemma 1}

The following geometric relations:

$$
\begin{gathered}
\left|\tilde{\Omega}_{n(\alpha)}^{k}\right|=\hat{K}^{k} \hat{C}_{\alpha}^{k} \hat{\boldsymbol{l}}_{\alpha}^{k} \cdot \hat{\boldsymbol{s}}_{\alpha}^{k}, \\
\sum_{1}^{3 n^{k}} \hat{p}_{\alpha i}^{k} \hat{K}^{k} \hat{C}_{\alpha}^{k} \hat{\boldsymbol{l}}_{\alpha}^{k}=\tilde{\boldsymbol{l}}_{i}^{k}
\end{gathered}
$$

hold.

\section{Proof}

The thesis stems from the following purely geometric identities. For an (oblique) parallelepiped we have that

$$
\begin{aligned}
\left(\hat{K}^{k} \hat{C}_{\alpha}^{k}\right)\left(\hat{\boldsymbol{l}}_{\alpha}^{k} \cdot \hat{\boldsymbol{s}}_{\alpha}^{k}\right) & =\frac{1}{8} 8\left|\tilde{\boldsymbol{\Omega}}_{n(\alpha)}^{k}\right| \\
\sum_{1}^{3 n^{k}}\left(\hat{K}^{k} \hat{C}_{\alpha}^{k}\right)\left(\hat{p}_{\alpha i}^{k} \hat{\boldsymbol{l}}_{\alpha}^{k}\right) & =\frac{1}{8} \sum_{1}^{3 n^{k}} \hat{p}_{\alpha i}^{k} \hat{\boldsymbol{l}}_{\alpha}^{k}=\frac{1}{8} 8 \tilde{\boldsymbol{l}}_{i}^{k}
\end{aligned}
$$

hold. For an (oblique) triangular prism (when $\hat{\Gamma}_{\alpha}^{k}$ is a parallelogram), we have that

$$
\begin{aligned}
\left(\hat{K}^{k} \hat{C}_{\alpha}^{k}\right)\left(\hat{\boldsymbol{l}}_{\alpha}^{k} \cdot \hat{\boldsymbol{s}}_{\alpha}^{k}\right) & =\frac{1}{6} 6\left|\tilde{\Omega}_{n(\alpha)}^{k}\right|, \\
\sum_{1}^{3 n^{k}}\left(\hat{K}^{k} \hat{C}_{\alpha}^{k}\right)\left(\hat{p}_{\alpha i}^{k} \hat{\boldsymbol{l}}_{\alpha}^{k}\right) & =\frac{1}{6} \sum_{1}^{3 n^{k}} \hat{p}_{\alpha i}^{k} \hat{\boldsymbol{l}}_{\alpha}^{k}=\frac{1}{6} 6 \tilde{\boldsymbol{l}}_{i}^{k} .
\end{aligned}
$$

hold. For an (oblique) triangular prism (when $\hat{\Gamma}_{\alpha}^{k}$ is a triangle) and for a tetrahedron we have that

$$
\begin{aligned}
\left(\hat{K}^{k} \hat{C}_{\alpha}^{k}\right)\left(\hat{\boldsymbol{l}}_{\alpha}^{k} \cdot \hat{\boldsymbol{s}}_{\alpha}^{k}\right) & =\frac{1}{12} 12\left|\tilde{\Omega}_{n(\alpha)}^{k}\right| \\
\sum_{1}^{3 n^{k}}\left(\hat{K}^{k} \hat{\boldsymbol{C}}_{\alpha}^{k}\right)\left(\hat{p}_{\alpha i}^{k} \hat{\boldsymbol{l}}_{\alpha}^{k}\right) & =\frac{1}{12} \sum_{1}^{3 n^{k}} \alpha \hat{p}_{\alpha i}^{k} \hat{\boldsymbol{l}}_{\alpha}^{k}=\frac{1}{12} 12 \tilde{\boldsymbol{l}}_{i}^{k} .
\end{aligned}
$$

hold. 
The following step-wise uniform vector functions attached to the face $\hat{\Sigma}_{\alpha}$ are now introduced:

$$
\hat{\boldsymbol{w}}_{\alpha}^{k}(\boldsymbol{r})= \begin{cases}\frac{\hat{K}^{k} \hat{C}_{\alpha}^{k} \hat{\boldsymbol{l}}_{\alpha}^{k}}{\left|\tilde{\Omega}_{n(\alpha)}^{k}\right|}, & \boldsymbol{r} \in \tilde{\Omega}_{n(\alpha)}^{k}, \\ \mathbf{0}, & \boldsymbol{r} \notin \tilde{\Omega}_{n(\alpha)}^{k} .\end{cases}
$$

\section{Lemma 2}

The $\hat{\boldsymbol{w}}_{\alpha}^{k}(\boldsymbol{r})$ vector functions satisfy the following properties:

$$
\begin{aligned}
\int_{\hat{\Sigma}_{\alpha}^{k} \cap \tilde{\Omega}_{n(\alpha)}^{k}} \hat{\boldsymbol{w}}_{\beta}^{k}(\boldsymbol{r}) \cdot \boldsymbol{n}(\boldsymbol{r}) \mathrm{d} \Sigma & =\frac{\left|\hat{\Sigma}_{\alpha}^{k} \cap \tilde{\Omega}_{n(\alpha)}^{k}\right|}{\left|\hat{\Sigma}_{\alpha}^{k}\right|} \delta_{\alpha \beta}, \quad \alpha, \beta=1, \ldots, 3 n^{k}, \\
\sum_{1}^{3 n_{\alpha}^{k}} \hat{\boldsymbol{w}}_{\alpha}^{k}(\boldsymbol{r}) \otimes \hat{\boldsymbol{s}}_{\alpha}^{k} & =\boldsymbol{I}, \\
\hat{K}^{k} \hat{C}_{\alpha}^{k} \hat{\boldsymbol{l}}_{\alpha}^{k} & =\int_{\Omega^{k}} \hat{\boldsymbol{w}}_{\alpha}^{k}(\boldsymbol{r}) \mathrm{d} \Omega, \quad \alpha=1, \ldots, 3 n^{k} .
\end{aligned}
$$

\section{Proof}

If $n(\alpha) \neq n(\beta)$, since $\tilde{\Omega}_{n(\alpha)}^{k}$ and $\tilde{\Omega}_{n(\beta)}^{k}$ are disjoint, the left-hand side of (20) is zero. Otherwise if $n(\alpha)=n(\beta)$ then

$$
\int_{\hat{\Sigma}_{\alpha}^{k} \cap \tilde{\Omega}_{n(\alpha)}^{k}} \hat{\boldsymbol{w}}_{\beta}^{k}(\boldsymbol{r}) \cdot \boldsymbol{n}(\boldsymbol{r}) \mathrm{d} \Gamma=\frac{\left|\hat{\Sigma}_{\alpha}^{k} \cap \tilde{\boldsymbol{\Omega}}_{n(\alpha)}^{k}\right|}{\left|\hat{\Sigma}_{\alpha}^{k}\right|} \hat{\boldsymbol{s}}_{\alpha}^{k} \cdot \frac{\hat{K}^{k} \hat{\boldsymbol{C}}_{\beta}^{k} \hat{\boldsymbol{l}}_{\beta}^{k}}{\left|\tilde{\boldsymbol{\Omega}}_{n(\beta)}^{k}\right|}
$$

which is zero if $\alpha \neq \beta$, since $\hat{\boldsymbol{l}}_{\alpha}^{k} \cdot \hat{\boldsymbol{s}}_{\beta}^{k}=0$, and is $\left|\hat{\Sigma}_{\alpha}^{k} \cap \tilde{\Omega}_{n(\alpha)}^{k}\right| /\left|\hat{\Sigma}_{\alpha}^{k}\right|$, as a consequence of Lemma 1 , if $\alpha=\beta$. Thus (20) is proved.

Let $\boldsymbol{r} \in \tilde{\Omega}_{i}^{k}$. The sum in the left-hand side of (21) has only three non-zero terms for the values $\alpha_{1}, \alpha_{2}, \alpha_{3}$ of the index $\alpha$ such that $n\left(\alpha_{1}\right)=n\left(\alpha_{2}\right)=n\left(\alpha_{3}\right)=i$. Thus, from the definition of $\hat{\boldsymbol{w}}_{\alpha}^{k}(\boldsymbol{r})$, from Lemma 1, and since $\hat{\boldsymbol{s}}_{\alpha_{1}}^{k}, \hat{\boldsymbol{s}}_{\alpha_{2}}^{k}, \hat{\boldsymbol{s}}_{\alpha_{3}}^{k}$ are parallel to $\hat{\boldsymbol{l}}_{\alpha_{2}}^{k} \times \hat{\boldsymbol{l}}_{\alpha_{3}}^{k}, \hat{\boldsymbol{l}}_{\alpha_{3}}^{k} \times \hat{\boldsymbol{l}}_{\alpha_{1}}^{k}, \hat{\boldsymbol{l}}_{\alpha_{1}}^{k} \times \hat{\boldsymbol{l}}_{\alpha_{2}}^{k}$, respectively, it follows:

$$
\begin{aligned}
\sum_{1}^{3 n^{k}} \hat{\boldsymbol{w}}_{\alpha}^{k}(\boldsymbol{r}) \otimes \hat{\boldsymbol{s}}_{\alpha}^{k}= & \frac{\hat{\boldsymbol{l}}_{\alpha_{1}}^{k} \otimes \hat{\boldsymbol{s}}_{\alpha_{1}}^{k}}{\hat{\boldsymbol{\boldsymbol { s }}}_{\alpha_{1}}^{k} \cdot \hat{\boldsymbol{l}}_{\alpha_{1}}^{k}}+\frac{\hat{\boldsymbol{l}}_{\alpha_{2}}^{k} \otimes \hat{\boldsymbol{s}}_{\alpha_{2}}^{k}}{\hat{\boldsymbol{s}}_{\alpha_{1}}^{k} \cdot \hat{\boldsymbol{l}}_{\alpha_{2}}^{k}}+\frac{\hat{\boldsymbol{l}}_{\alpha_{3}}^{k} \otimes \hat{\boldsymbol{s}}_{\alpha_{3}}^{k}}{\hat{\boldsymbol{s}}_{\alpha_{3}}^{k} \cdot \hat{\boldsymbol{l}}_{\alpha_{3}}^{k}} \\
& =\frac{\left(\hat{\boldsymbol{l}}_{\alpha_{2}}^{k} \times \hat{\boldsymbol{l}}_{\alpha_{3}}^{k}\right) \otimes \hat{\boldsymbol{l}}_{\alpha_{1}}^{k}}{\left(\hat{\boldsymbol{l}}_{\alpha_{2}}^{k} \times \hat{\boldsymbol{l}}_{\alpha_{3}}^{k}\right) \cdot \hat{\boldsymbol{l}}_{\alpha_{1}}^{k}}+\frac{\left(\hat{\boldsymbol{l}}_{\alpha_{3}}^{k} \times \hat{\boldsymbol{l}}_{\alpha_{1}}^{k}\right) \otimes \hat{\boldsymbol{l}}_{\alpha_{2}}^{k}}{\left(\hat{\boldsymbol{l}}_{\alpha_{3}}^{k} \times \hat{\boldsymbol{l}}_{\alpha_{1}}^{k}\right) \cdot \hat{\boldsymbol{l}}_{\alpha_{2}}^{k}}+\frac{\left(\hat{\boldsymbol{l}}_{\alpha_{1}}^{k} \times \hat{\boldsymbol{l}}_{\alpha_{2}}^{k}\right) \otimes \hat{\boldsymbol{l}}_{\alpha_{3}}^{k}}{\left(\hat{\boldsymbol{l}}_{\alpha_{1}}^{k} \times \hat{\boldsymbol{l}}_{\alpha_{2}}^{k}\right) \cdot \hat{\boldsymbol{l}}_{\alpha_{3}}^{k}}=\boldsymbol{I}
\end{aligned}
$$

in which the last equality for $\hat{\boldsymbol{l}}_{\alpha_{1}}^{k}, \hat{\boldsymbol{l}}_{\alpha_{2}}^{k}, \hat{\boldsymbol{l}}_{\alpha_{3}}^{k}$ can be directly verified, and (21) is proved. 
Lastly, by recalling the definition of $\hat{\boldsymbol{w}}_{\alpha}^{k}(\boldsymbol{r}),(22)$ straightforwardly descends.

\subsection{The face vector functions and their properties}

The following result can now be proved.

Theorem 1

The vector functions attached to the face $\Sigma_{i}^{k}$

$$
\boldsymbol{w}_{i}^{k}(\boldsymbol{r})=\sum_{1}^{3 n^{k}} \hat{\boldsymbol{w}}_{\alpha}^{k}(\boldsymbol{r}) \hat{p}_{\alpha i}^{k}, \quad i=1, \ldots, f^{k}
$$

satisfy the following geometric properties:

$$
\begin{aligned}
\int_{\Sigma_{j}^{k}} \boldsymbol{w}_{i}^{k}(\boldsymbol{r}) \cdot \boldsymbol{n}(\boldsymbol{r}) \mathrm{d} \Sigma & =\delta_{i j}, \quad i, j=1, \ldots, f^{k}, \\
\sum_{1}^{f_{i}^{k}} \boldsymbol{w}_{i}^{k}(\boldsymbol{r}) \otimes \boldsymbol{s}_{i}^{k} & =\boldsymbol{I}, \\
\int_{\Omega} \boldsymbol{w}_{i}^{k}(\boldsymbol{r}) \mathrm{d} \Omega & =\tilde{\boldsymbol{l}}_{i}^{k}, \quad i=1, \ldots, f^{k} .
\end{aligned}
$$

Proof

From the definition of $\boldsymbol{w}_{i}^{k}(\boldsymbol{r})$ it follows:

$$
\begin{aligned}
\int_{\Sigma_{j}^{k}} \boldsymbol{w}_{i}^{k}(\boldsymbol{r}) \cdot \boldsymbol{n}(\boldsymbol{r}) \mathrm{d} \Sigma & =\sum_{1}^{3 n^{k}} \hat{p}_{\alpha i}^{k} \int_{\Sigma_{j}^{k}} \hat{\boldsymbol{w}}_{\alpha}^{k}(\boldsymbol{r}) \cdot \boldsymbol{n}(\boldsymbol{r}) \mathrm{d} \Sigma \\
& =\sum_{1}^{3 n^{k}} \alpha \hat{p}_{\alpha i}^{k} \sum_{1}^{3 n^{k}} \beta \int_{\hat{\Sigma}_{\beta}^{k} \cap \tilde{\Omega}_{n(\beta)}^{k}} \hat{\boldsymbol{w}}_{\alpha}^{k}(\boldsymbol{r}) \cdot \boldsymbol{n}(\boldsymbol{r}) \mathrm{d} \Sigma \\
& =\sum_{1}^{3 n^{k}} \alpha \hat{p}_{\alpha i}^{k} \sum_{1}^{3 n^{k}} \hat{p}_{\beta j}^{k} \int_{\hat{\Sigma}_{\beta}^{k} \cap \tilde{\Omega}_{n(\beta)}^{k}} \hat{\boldsymbol{w}}_{\alpha}^{k}(\boldsymbol{r}) \cdot \boldsymbol{n}(\boldsymbol{r}) \mathrm{d} \Sigma \\
& =\sum_{1}^{3 n^{k}} \alpha \beta \hat{p}_{\alpha i}^{k} \hat{p}_{\beta j}^{k} \frac{\left|\hat{\Sigma}_{\alpha}^{k} \cap \tilde{\Omega}_{n(\alpha)}^{k}\right|}{\left|\hat{\Sigma}_{\alpha}^{k}\right|} \delta_{\alpha \beta}, \\
& =\sum_{1}^{3 n^{k}} \frac{\left|\hat{\Sigma}_{\alpha}^{k} \cap \tilde{\Omega}_{n(\alpha)}^{k}\right|}{\left|\hat{\Sigma}_{\alpha}^{k}\right|} \hat{p}_{\alpha i}^{k} \hat{p}_{\alpha j}^{k}=\delta_{i j},
\end{aligned}
$$

Equation (26) is descending from (20). Equation (23) is thus proved. 
From the definition of $\boldsymbol{w}_{i}^{k}(\boldsymbol{r})$ and from (21) it follows:

$$
\begin{aligned}
\sum_{1}^{f_{i}^{k}} \boldsymbol{w}_{i}^{k}(\boldsymbol{r}) \otimes \boldsymbol{s}_{i}^{k} & =\sum_{1}^{f^{k}} \sum_{1}^{3 n^{k}}{ }_{\alpha} \hat{\boldsymbol{w}}_{\alpha}^{k}(\boldsymbol{r}) \hat{t}_{\alpha i}^{k} \otimes \boldsymbol{s}_{i}^{k} \\
& =\sum_{1}^{3 n^{k}} \hat{\boldsymbol{w}}_{\alpha}^{k}(\boldsymbol{r}) \otimes \sum_{1}^{f_{i}} \hat{p}_{\alpha i}^{k} \boldsymbol{s}_{i}^{k} \\
& =\sum_{1}^{3 n^{k}} \hat{\boldsymbol{w}}_{\alpha}^{k}(\boldsymbol{r}) \otimes \hat{\boldsymbol{s}}_{\alpha}^{k}=\boldsymbol{I} .
\end{aligned}
$$

Equation (24) is thus proved.

Lastly from the definition of $\boldsymbol{w}_{i}^{k}(\boldsymbol{r})$, from (22) and from (18) it follows:

$$
\begin{aligned}
\int_{\Omega} \boldsymbol{w}_{i}^{k}(\boldsymbol{r}) \mathrm{d} \Omega & =\int_{\Omega} \sum_{1}^{3 n^{k}} \hat{\boldsymbol{w}}_{\alpha}^{k}(\boldsymbol{r}) \hat{p}_{\alpha i}^{k} \mathrm{~d} \Omega \\
& =\sum_{1}^{3 n^{k}} \alpha \hat{p}_{\alpha i}^{k} \int_{\Omega} \hat{\boldsymbol{w}}_{\alpha}^{k}(\boldsymbol{r}) \mathrm{d} \Omega \\
& =\sum_{1}^{3 n^{k}} \hat{p}_{\alpha i}^{k} \hat{K}^{k} \hat{C}_{\alpha}^{k} \hat{\boldsymbol{l}}_{\alpha}^{k}=\tilde{\boldsymbol{l}}_{i}^{k}, \quad i=1, \ldots, f^{k} .
\end{aligned}
$$

Equation (25) is thus proved.

We will now introduce the current density field computed in $\Omega^{k}$ from the currents $i_{i}^{k}(t)$ across the faces $\mathscr{G}^{k}$. We denote with $\pi_{f}^{k}(\boldsymbol{r}) \mathbf{i}^{k}(t)$ such current density field, where symbol $\pi_{f}^{k}(\boldsymbol{r})$ acts on the fluxes across the faces (currents in the specific case) of $\mathscr{G}^{k}$ yielding a vector field (a computed current density vector in the specific case). Of course the current density vector $\pi_{f}^{k}(\boldsymbol{r}) \mathbf{i}^{k}(t)$ only approximates the actual current density vector $\boldsymbol{j}(\boldsymbol{r}, t)$. We demonstrate the following theorem.

\section{Theorem 2}

The functions $\boldsymbol{w}_{i}^{k}(\boldsymbol{r})$ with $i=1, \ldots, f^{k}$ form a basis and for the current density field

$$
\boldsymbol{\pi}_{f}^{k}(\boldsymbol{r}) \mathbf{i}^{k}(t)=\sum_{1}^{f^{k}} i_{i}^{k}(t) \boldsymbol{w}_{i}^{k}(\boldsymbol{r}),
$$

the degrees of freedom $i_{i}^{k}(t)$ with $i=1, \ldots, f^{k}$ are the fluxes of $\pi_{f}^{k}(\boldsymbol{r}) \mathbf{i}^{k}(t)$ through the faces of $\mathscr{G}^{k}$.

\section{Proof}

By computing the fluxes of $\pi_{f}^{k}(\boldsymbol{r}) \mathbf{i}^{k}(t)$ through the faces $\Sigma_{j}^{k}$, with $j=1, \ldots, f^{k}$, and by using (23) it results in

$$
\begin{aligned}
\int_{\Sigma_{j}^{k}} \pi_{f}^{k}(\boldsymbol{r}) \mathbf{i}^{k}(t) \cdot \boldsymbol{n}(\boldsymbol{r}) \mathrm{d} \Sigma & =\sum_{1}^{f_{i}^{k}} i_{i}^{k}(t) \int_{\Sigma_{j}^{k}} \boldsymbol{w}_{i}^{k}(\boldsymbol{r}) \cdot \boldsymbol{n}(\boldsymbol{r}) \mathrm{d} \Sigma \\
& =\sum_{1}^{f_{i}^{k}} i_{i}^{k}(t) \delta_{i j}=i_{j}^{k}(t), \quad j=1, \ldots, f^{k} .
\end{aligned}
$$


Thus, the degrees of freedom $i_{j}^{k}(t)$ are the fluxes of $\pi_{f}^{k}(\boldsymbol{r}) \mathbf{i}^{k}(t)$ through $\Sigma_{j}^{k}$, with $i=j, \ldots, f^{k}$. Besides if $\pi_{f}^{k}(\boldsymbol{r}) \mathbf{i}^{k}(t)=\mathbf{0}$ then from (27) it follows $i_{j}^{k}(t)=0$, with $j=1, \ldots, f^{k}$. Thus, vector functions $\boldsymbol{w}_{i}^{k}(\boldsymbol{r})$, with $i=1, \ldots, f^{k}$, form a basis.

Theorem 3

Field $\pi_{f}^{k}(\boldsymbol{r}) \mathbf{i}^{k}(t)$ encompasses all fields spatially uniform in $\Omega^{k}$.

Proof

By applying both members of (24) to a uniform vector $\boldsymbol{a}$, it results in

$$
\boldsymbol{a}=\left(\sum_{1}^{f_{i}^{k}} \boldsymbol{w}_{i}^{k}(\boldsymbol{r}) \otimes \boldsymbol{s}_{i}^{k}\right) \boldsymbol{a}=\sum_{1}^{f_{i}^{k}}\left(\boldsymbol{a} \cdot \boldsymbol{s}_{i}^{k}\right) \boldsymbol{w}_{i}^{k}(\boldsymbol{r})=\sum_{1}^{f_{i}^{k}} A_{i}^{k} \boldsymbol{w}_{i}^{k}(\boldsymbol{r}),
$$

in which

$$
A_{i}^{k}=\int_{\Sigma_{i}^{k}} \boldsymbol{a} \cdot \boldsymbol{n}(\boldsymbol{r}) \mathrm{d} \Sigma, \quad i=1, \ldots, f^{k} .
$$

and the thesis follows.

As a consequence, the $\boldsymbol{w}_{i}^{k}(\boldsymbol{r})$ vector functions can be used as a basis for representing vector fields within $\Omega^{k}$ which can be locally approximated as uniform. Such functions are different from the face element vector functions used in the Finite Element Method (FEM). The vector field $\pi_{f}^{k}(\boldsymbol{r}) \mathbf{i}^{k}(t)$ will be used as an approximation of the current density field $\boldsymbol{j}(\boldsymbol{r}, t)$ within $\Omega^{k}$.

\section{THE CONSTITUTIVE MATRIX E}

Let $\boldsymbol{\eta}^{k}(\boldsymbol{r})$ be the restriction of the $\boldsymbol{\eta}(\boldsymbol{r})$ resistivity tensor to the region $\Omega^{k}$. Let $\mathbf{i}^{k}(t)$ be the $f^{k} \times 1$ array of the approximations of the fluxes $i_{i}^{k}(t)$, with $i=1, \ldots, f^{k}$, of the current density $\boldsymbol{j}(\boldsymbol{r}, t)$ through the faces of $\mathscr{G}^{k}$. Let $\tilde{\mathbf{v}}^{k}(t)$ be the $f^{k} \times 1$ array of the approximations of the circulations $\tilde{v}_{i}^{k}(t)$ of the electric field $\boldsymbol{e}(\boldsymbol{r}, t)$ along the along the edges of $\tilde{\mathscr{G}}^{k}$.

If $\boldsymbol{e}(\boldsymbol{r}, t)$ is uniform in $\Omega^{k}$ then from (25) it is, for $i=1 \ldots, \tilde{l}^{k}$,

$$
\int_{\tilde{\Gamma}_{i}^{k}} \boldsymbol{e}(\boldsymbol{r}, t) \mathrm{d} \Gamma_{\boldsymbol{r}}=\int_{\Omega^{k}} \boldsymbol{e}(\boldsymbol{r}, t) \cdot \boldsymbol{w}_{i}^{k}(\boldsymbol{r}) \mathrm{d} \Omega_{\boldsymbol{r}}
$$

Thus if $\boldsymbol{e}(\boldsymbol{r}, t)$ can be approximated as locally uniform, then the following relations can be assumed for approximating $\tilde{v}_{i}^{k}(t)$, with $i=1 \ldots, \tilde{l}^{k}$,

$$
\tilde{v}_{i}^{k}(t)=\int_{\Omega^{k}} \boldsymbol{e}(\boldsymbol{r}, t) \cdot \boldsymbol{w}_{i}^{k}(\boldsymbol{r}) \mathrm{d} \Omega_{\boldsymbol{r}}
$$

Besides if $\boldsymbol{j}(\boldsymbol{r}, t)$ can be approximated as locally uniform then it can be represented in each $\Omega^{k}$ by $\pi_{f}^{k}(\boldsymbol{r}) \mathbf{i}^{k}(t)$ and if the resistivity tensor $\boldsymbol{\eta}^{k}(\boldsymbol{r})$ can be approximated as locally homogeneous it can be 
approximated by its value $\boldsymbol{\eta}^{k}\left(\boldsymbol{r}^{k}\right)$ evaluated at the node $\boldsymbol{r}^{k}$. Thus, from Ohm's constitutive relation and Theorem 2 it results in

$$
\boldsymbol{e}(\boldsymbol{r}, t)=\boldsymbol{\eta}^{k}\left(\boldsymbol{r}^{k}\right) \sum_{1}^{f^{k}} i_{j}^{k}(t) \boldsymbol{w}_{j}^{k}(\boldsymbol{r})
$$

By substituting (29) into (28) we obtain for $\tilde{v}_{i}^{k}(t)$, with $i=1 \ldots, \tilde{l}^{k}$, the following equation:

$$
\tilde{v}_{i}^{k}(t)=\sum_{1}^{f^{k}}\left(\int_{\Omega^{k}} \boldsymbol{w}_{i}^{k}(\boldsymbol{r}) \cdot \boldsymbol{\eta}^{k}\left(\boldsymbol{r}^{k}\right) \boldsymbol{w}_{j}^{k}(\boldsymbol{r}) \mathrm{d} \Omega_{\boldsymbol{r}}\right) i_{j}^{k}
$$

Equivalently

$$
\mathbf{v}^{k}(t)=\mathbf{E}^{k} \mathbf{i}^{k}(t)
$$

in which $\mathbf{E}^{k}$ is an $f^{k} \times f^{k}$ matrix whose elements are

$$
E_{i j}^{k}=\int_{\Omega^{k}} \boldsymbol{w}_{i}^{k}(\boldsymbol{r}) \cdot \boldsymbol{\eta}^{k}\left(\boldsymbol{r}^{k}\right) \boldsymbol{w}_{j}^{k}(\boldsymbol{r}) \mathrm{d} \Omega, \quad i, j=1, \ldots, f^{k},
$$

By construction, if $\boldsymbol{\eta}(\boldsymbol{r}), \boldsymbol{e}(\boldsymbol{r}, t), \boldsymbol{j}(\boldsymbol{r}, t)$ are spatially uniform, then (31), (32) are exact equations. Thus if $\boldsymbol{\eta}(\boldsymbol{r}), \boldsymbol{e}(\boldsymbol{r}, t), \boldsymbol{j}(\boldsymbol{r}, t)$ can be approximated as locally homogeneous, then (31), (32) can be assumed as an approximate equation relating $\mathbf{i}^{k}(t), \tilde{\mathbf{v}}^{k}(t)$ with $k=1, \ldots, v$.

Besides the following property for $\mathbf{E}^{k}$ descends.

\section{Lemma 3}

Matrix $\mathbf{E}^{k}$ is symmetric, positive-definite.

Proof

Since the resistivity tensor $\boldsymbol{\eta}^{k}\left(\boldsymbol{r}^{k}\right)$ is symmetric, it follows:

$$
E_{i j}^{k}=\int_{\Omega^{k}} \boldsymbol{w}_{i}^{k}(\boldsymbol{r}) \cdot \boldsymbol{\eta}^{k}\left(\boldsymbol{r}^{k}\right) \boldsymbol{w}_{j}^{k}(\boldsymbol{r}) \mathrm{d} \boldsymbol{\Omega}=\int_{\Omega^{k}} \boldsymbol{w}_{j}^{k}(\boldsymbol{r}) \cdot \boldsymbol{\eta}^{k}\left(\boldsymbol{r}^{k}\right) \boldsymbol{w}_{i}^{k}(\boldsymbol{r}) \mathrm{d} \Omega=E_{j i}^{k} \quad i, j=1, \ldots, f^{k}
$$

and $\mathbf{E}^{k}$ is symmetric. Since the resistivity tensor $\boldsymbol{\eta}^{k}\left(\boldsymbol{r}^{k}\right)$ is positive-definite, it follows:

$$
\begin{aligned}
\mathbf{i}^{k}(t){ }^{\mathrm{T}} \mathbf{E}^{k} \mathbf{i}^{k}(t) & =\sum_{1}^{k} i_{j} i_{i}^{k}(t)\left(\int_{\Omega^{k}} \boldsymbol{w}_{i}^{k}(\boldsymbol{r}) \cdot \boldsymbol{\eta}^{k}\left(\boldsymbol{r}^{k}\right) \boldsymbol{w}_{j}^{k}(\boldsymbol{r}) \mathrm{d} \Omega\right) i_{j}^{k}(t) \\
& =\int_{\Omega^{k}}\left(\sum_{1}^{f_{i}} i_{i}^{k}(t) \boldsymbol{w}_{i}^{k}(\boldsymbol{r})\right) \cdot \boldsymbol{\eta}^{k}\left(\boldsymbol{r}^{k}\right)\left(\sum_{1}^{f_{j}^{k}} i_{j}^{k}(t) \boldsymbol{w}_{j}^{k}(\boldsymbol{r})\right) \mathrm{d} \Omega \\
& =\int_{\Omega^{k}} \pi_{f}^{k}(\boldsymbol{r}) \mathbf{i}^{k}(t) \cdot \boldsymbol{\eta}^{k}\left(\boldsymbol{r}^{k}\right) \pi_{f}^{k}(\boldsymbol{r}) \tilde{\mathbf{i}}^{k}(t) \mathrm{d} \boldsymbol{\Omega} \geqslant 0
\end{aligned}
$$

and $\mathbf{E}^{k}$ is positive semi-definite. Besides $\mathbf{i}^{k}(t)^{\mathrm{T}} \mathbf{E}^{k} \mathbf{i}^{k}(t)=0$ implies $\pi_{f}^{k}(\boldsymbol{r}) \mathbf{i}^{k}(t)=\mathbf{0}$ and, from Lemma 2 , also $\mathbf{i}^{k}(t)=\mathbf{0}$. Thus, $\mathbf{E}^{k}$ is positive-definite. 


\subsection{From local $\mathbf{E}^{k}$ to resistance matrix $\mathbf{E}$}

The resistance $\mathbf{E}$ matrix is now generated as follows. Let $\mathbf{P}^{k}$ be the $f^{k} \times f$ matrix whose element $p_{i j}^{k}$ is 1 if the $i$ th face of $\mathscr{G}^{k}$ is the $j$ th face of $\mathscr{G}$ and is 0 otherwise. Then it is

$$
\mathbf{i}^{k}(t)=\mathbf{P}^{k} \mathbf{i}(t) .
$$

Let it be

$$
\mathbf{E}={ }_{k} \sum_{1}^{v} \mathbf{P}^{k^{\mathrm{T}}} \mathbf{E}^{k} \mathbf{P}^{k}
$$

Since

$$
\mathbf{v}(t)=\sum_{1}^{v}{ }_{k} \mathbf{P}^{k \mathrm{~T}} \mathbf{v}^{k}(t)=\sum_{1}^{v}{ }_{k} \mathbf{P}^{k \mathrm{~T}} \mathbf{E}^{k} \mathbf{i}^{k}(t)=\sum_{1}^{v}{ }_{k} \mathbf{P}^{k \mathrm{~T}} \mathbf{E}^{k}\left(\mathbf{P}^{k} \mathbf{i}(t)\right)=\mathbf{E i}(t)
$$

then, from Lemma 3, E is symmetric, positive-definite, and it is a discrete counterpart of the $\boldsymbol{\eta}(\boldsymbol{r})$ tensor.

\section{EXPRESSION OF $\tilde{\mathbf{a}}(t)$ IN TERMS OF $\mathbf{i}(t)$}

If $\boldsymbol{A}(\boldsymbol{r}, t)$ is uniform in $\Omega^{k}$ then from (25) it is, for $i=1 \ldots, \tilde{l}^{k}$,

$$
\int_{\tilde{\Gamma}_{i}^{k}} \boldsymbol{A}(\boldsymbol{r}, t) \mathrm{d} \Gamma_{\boldsymbol{r}}=\int_{\Omega^{k}} \boldsymbol{A}(\boldsymbol{r}, t) \cdot \boldsymbol{w}_{i}^{k}(\boldsymbol{r}) \mathrm{d} \Omega_{\boldsymbol{r}}
$$

Thus if $\boldsymbol{A}(\boldsymbol{r}, t)$ can be approximated as locally uniform, the following relations can be assumed for approximating $\tilde{a}_{i}^{k}(t)$, with $i=1 \ldots, \tilde{l}^{k}$ :

$$
\tilde{a}_{i}^{k}(t)=\int_{\Omega^{k}} \boldsymbol{A}(\boldsymbol{r}, t) \cdot \boldsymbol{w}_{i}^{k}(\boldsymbol{r}) \mathrm{d} \Omega \boldsymbol{r}
$$

Besides, if $\boldsymbol{j}(\boldsymbol{r}, t)$ can be approximated as locally uniform then it can be represented in each $\Omega^{k}$ by $\pi_{f}^{k}(\boldsymbol{r}) \mathbf{i}^{k}(t)$. Thus from (4) it results in

$$
\boldsymbol{A}(\boldsymbol{r}, t)=\frac{\mu}{4 \pi} \sum_{1}^{v} \sum_{1}^{f^{k}} \sum_{j} i_{j}^{k}(t) \int_{\Omega^{k}} \frac{\boldsymbol{w}_{j}^{k}(\boldsymbol{p})}{|\boldsymbol{r}-\boldsymbol{p}|} \mathrm{d} \Omega_{\boldsymbol{p}}
$$

By substituting (35) into (34) we obtain for $\tilde{a}_{i}^{k}(t)$, with $i=1 \ldots, \tilde{l}^{k}$,

$$
\tilde{a}_{i}^{h}(t)=\frac{\mu}{4 \pi} \sum_{1}^{v} \sum_{1}^{f_{j}^{k}} \tilde{i}_{j}^{k}(t) \int_{\Omega^{h}} \int_{\Omega^{k}} \frac{\boldsymbol{w}_{i}^{h}(\boldsymbol{r}) \cdot \boldsymbol{w}_{j}^{k}(\boldsymbol{p})}{|\boldsymbol{r}-\boldsymbol{p}|} \mathrm{d} \Omega_{\boldsymbol{r}} \mathrm{d} \boldsymbol{\Omega}_{\boldsymbol{p}},
$$

which can be equivalently rewritten as

$$
\tilde{\mathbf{a}}^{h}(t)=\sum_{1}^{v}{ }_{k} \mathbf{M}^{h k_{i} k}(t),
$$


where the elements of the $f^{h} \times f^{k}$ matrix $\mathbf{M}^{h k}$ are

$$
M_{i j}^{h k}=\frac{\mu}{4 \pi} \int_{\Omega^{h}} \int_{\Omega^{k}} \frac{\boldsymbol{w}_{i}^{h}(\boldsymbol{r}) \cdot \boldsymbol{w}_{j}^{k}(\boldsymbol{p})}{|\boldsymbol{r}-\boldsymbol{p}|} \mathrm{d} \Omega_{\boldsymbol{r}} \mathrm{d} \Omega_{\boldsymbol{p}}
$$

with $i=1, \ldots, f^{h}, j=1, \ldots, f^{k}$.

By construction, if $\boldsymbol{A}(\boldsymbol{r}, t)$ and $\boldsymbol{j}(\boldsymbol{r}, t)$ are spatially uniform, then (37), (38) are exact equations. Thus if $\boldsymbol{A}(\boldsymbol{r}, t), \boldsymbol{j}(\boldsymbol{r}, t)$ can be approximated as locally uniform, then (37), (38) can be assumed as approximate equation relating $\mathbf{i}^{k}(t), \tilde{\mathbf{a}}^{k}(t)$ with $k=1, \ldots, v$.

The following property for $\mathbf{M}^{h k}$ descends.

\section{Lemma 4}

It results in

$$
\mathbf{M}^{h k \mathrm{~T}}=\mathbf{M}^{k h}
$$

Proof

It results in

$$
M_{i j}^{h k}=\frac{\mu}{4 \pi} \int_{\Omega^{h}} \int_{\Omega^{k}} \frac{\boldsymbol{w}_{i}^{h}(\boldsymbol{r}) \cdot \boldsymbol{w}_{j}^{k}(\boldsymbol{p})}{|\boldsymbol{r}-\boldsymbol{p}|} \mathrm{d} \Omega_{\boldsymbol{r}} \mathrm{d} \Omega_{\boldsymbol{p}}=\frac{\mu}{4 \pi} \int_{\Omega^{h}} \int_{\Omega^{k}} \frac{\boldsymbol{w}_{j}^{k}(\boldsymbol{r}) \cdot \boldsymbol{w}_{i}^{h}(\boldsymbol{p})}{|\boldsymbol{r}-\boldsymbol{p}|} \mathrm{d} \Omega_{\boldsymbol{r}} \mathrm{d} \Omega_{\boldsymbol{p}}=M_{j i}^{k h}
$$

and the claim follows.

\subsection{From local $\mathbf{M}^{h k}$ to global $\mathbf{M}$}

The global $\mathbf{M}$ matrix is now generated as follows. Let it be

$$
\mathbf{M}=\sum_{1}^{v} h \sum_{1}^{v}{ }_{k} \mathbf{P}^{h \mathrm{~T}} \mathbf{M}^{h k} \mathbf{P}^{k}
$$

Since

$$
\tilde{\mathbf{a}}(t)=\sum_{1}^{v}{ }_{h} \mathbf{P}^{h \mathrm{~T}} \tilde{\mathbf{a}}^{h}(t)
$$

holds. From (37), using (33), we obtain

$$
\tilde{\mathbf{a}}(t)=\sum_{1}^{v} h \sum_{1}^{v}{ }_{k} \mathbf{P}^{h \mathrm{~T}} \mathbf{M}^{h k}\left(\mathbf{P}^{k} \mathbf{i}(t)\right)=\mathbf{M i}(t) .
$$

Thus, $\mathbf{M}$ is a discrete counterpart of (4). Besides the following property descends for $\mathbf{M}$, which concurs in ensuring the stability of the discretized equations.

\section{Theorem 4}

Matrix $\mathbf{M}$ is symmetric, positive-definite. 
Proof

From Lemma 4 it results in

$$
\begin{aligned}
\mathbf{M}^{\mathrm{T}} & =\left(\sum_{1}^{v} h \sum_{1}^{v}{ }_{k} \mathbf{P}^{h \mathrm{~T}} \mathbf{M}^{h k} \mathbf{P}^{k}\right)^{\mathrm{T}} \\
& =\sum_{1}^{v} h \sum_{1}^{v}{ }_{k} \mathbf{P}^{k \mathrm{~T}} \mathbf{M}^{h k \mathrm{~T}} \mathbf{P}^{h}=\sum_{1}^{v} h_{h} \sum_{1}^{v}{ }_{k} \mathbf{P}^{k \mathrm{~T}} \mathbf{M}^{k h} \mathbf{P}^{h}=\mathbf{M}
\end{aligned}
$$

and $\mathbf{M}$ is symmetric. Besides it is

$$
\begin{aligned}
\mathbf{i}^{\mathrm{T}}(t) \mathbf{M i}(t) & =\sum_{1}^{v} h \sum_{1}^{v}{ }_{k} \mathbf{i}^{k}(t){ }^{\mathrm{T}} \mathbf{M}^{h k} \mathbf{i}^{k}(t) \\
& =\sum_{1}^{v} h \sum_{1}^{v}{ }_{k} \sum_{1}^{f_{i}} \sum_{1}^{f^{k}} i_{i}^{h}(t) i_{j}^{k}(t) \frac{\mu}{4 \pi} \int_{\Omega^{h}} \int_{\Omega^{k}} \frac{\boldsymbol{w}_{i}^{h}(\boldsymbol{r}) \cdot \boldsymbol{w}_{j}^{k}(\boldsymbol{p})}{|\boldsymbol{r}-\boldsymbol{p}|} \mathrm{d} \Omega_{\boldsymbol{r}} \mathrm{d} \Omega_{\boldsymbol{p}} \\
& =\frac{\mu}{4 \pi} \int_{\Omega_{\mathrm{c}}} \int_{\Omega_{\mathrm{c}}} \frac{\pi_{f}(\boldsymbol{r}) \mathbf{i}(t) \cdot \pi_{f}(\boldsymbol{p}) \mathbf{i}(t)}{|\boldsymbol{r}-\boldsymbol{p}|} \mathrm{d} \Omega_{r} \mathrm{~d} \Omega_{\boldsymbol{p}} \geqslant 0
\end{aligned}
$$

in which

$$
\pi_{f}(\boldsymbol{r}) \mathbf{i}(t)=\pi_{f}^{k}(\boldsymbol{r}) \mathbf{i}^{k}(t) \quad \text { for } \boldsymbol{r} \in \Omega^{k}
$$

and $\mathbf{M}$ is positive semi-definite; the last inequality descends from the known result [24] that magnetic energy functional is non-negative. Besides $\mathbf{i}(t)^{\mathrm{T}} \mathbf{M i}(t)=0$ implies $\pi_{f}(\boldsymbol{r}) \mathbf{i}(t)=\mathbf{0}$ and, from Lemma 2, also $\mathbf{i}(t)=\mathbf{0}$; thus $\mathbf{M}$ is positive-definite.

\section{NUMERICAL RESULTS}

As a numerical example, we considered the computation of eddy currents in a conducting aluminium plate (resistivity $\rho=2.5 \times 10^{-8} \Omega \mathrm{m}$ ) driven by a coil placed above it with a known impressed current density such that the overall coil current is $400 \mathrm{~A}$; the conducting domain has a hole drilled in it, Figure 2(a). We solved the eddy-current problem in the conducting domain $\Omega_{\mathrm{c}}$ with the proposed geometric integral formulation as a time harmonic problem at a frequency $f=5 \mathrm{kHz}$; the grid $\mathscr{G}$ in $\Omega_{\mathrm{c}}$ consists of 1708 nodes, 10044 edges and 7501 tetrahedra, Figure 2(b); to solve the final full complex system, we used the parallel library ScaLAPACK within the Intel Math Kernel Library (MKL). Figure 3 shows the real and imaginary parts of the amplitude of eddy-current density vector in the conductor, computed along a line $l$ with the geometric integral formulation; for comparison, a 2D reference finite element solution has been performed and the corresponding reference real and imaginary parts of the current density along the same line $l$ are drawn (solid lines) in addition.

\section{CONCLUSIONS}

We showed how the integral formulation of an eddy-current problem can be discretized according to the Discrete Geometric Approach. We rely on a purely geometric definition of a novel set of 

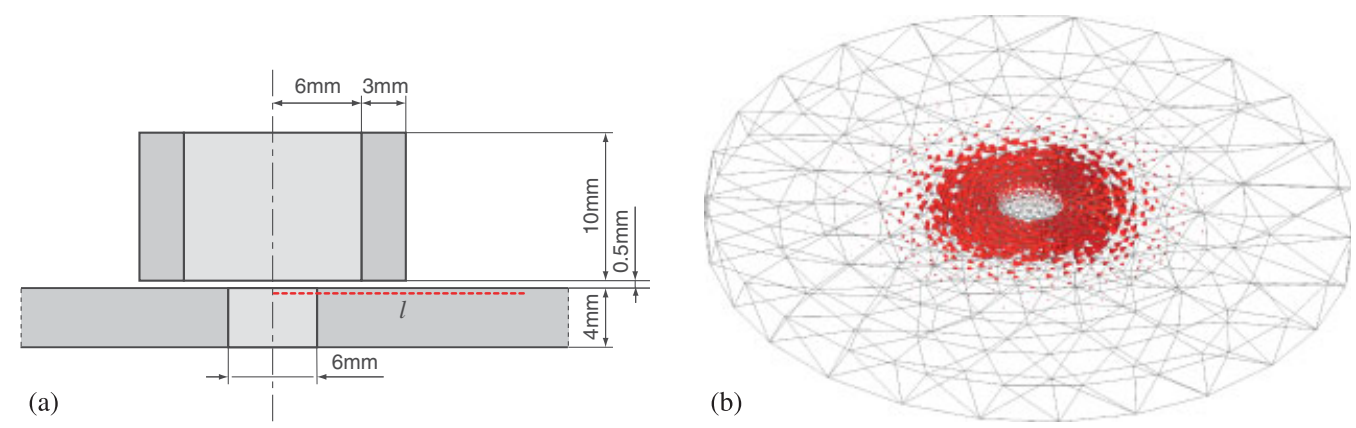

Figure 2. (a) Cross-section geometry of conducting and source regions; additionally line $l$ is shown. (b) The grid $\mathscr{G}$ in $\Omega_{\mathrm{c}}$ is shown and it consists of 1708 nodes, 10044 edges and 7501 tetrahedra. The eddy-current density vectors in $\Omega_{\mathrm{c}}$ are displayed also.
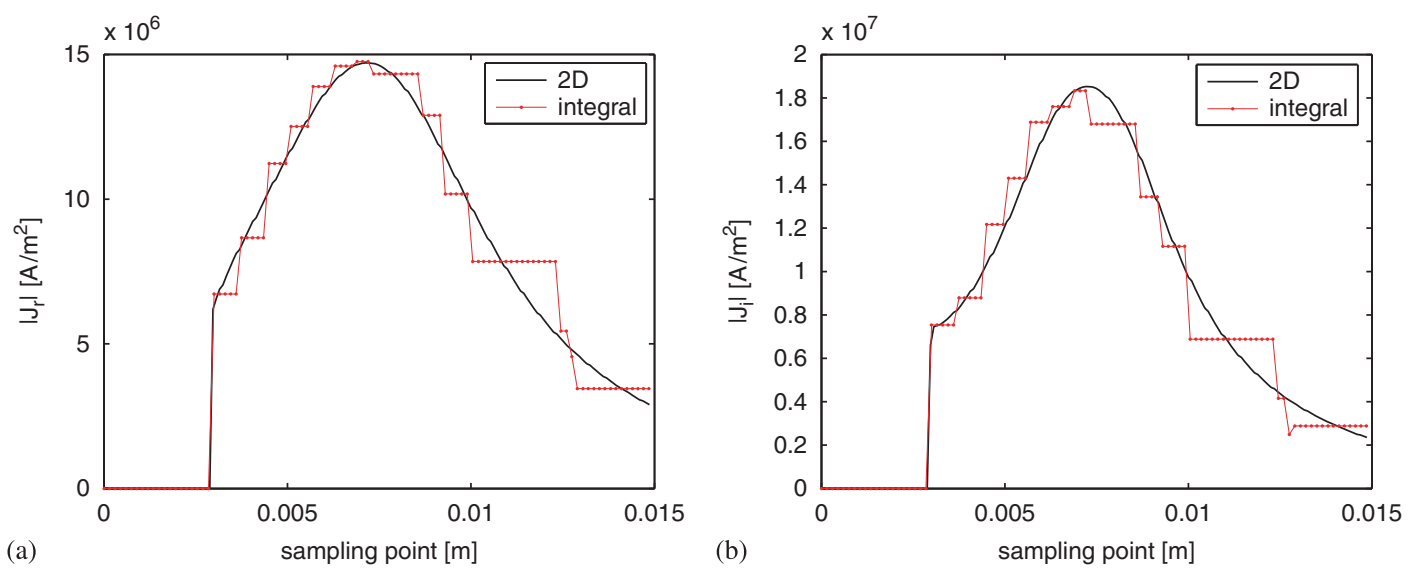

Figure 3. Real (left side) and imaginary (right side) parts of the amplitude of eddy-current density vector compared with respect to corresponding reference 2D finite element solutions (solid lines).

face vector basis functions that we used to construct the discrete counterparts-matrices-of both Ohm's constitutive relation and of the integral relation between the magnetic vector potential and the eddy-current density vector. The symmetry and positive-definiteness of such matrices have been proved and their entries are computed directly in terms of edge vectors associated with the edges of the mesh.

\section{REFERENCES}

1. Clemens M, Weiland T. Discrete electromagnetism with the finite integration technique. In PIER 32, Teixeira FL (ed.). EMW Publishing: Cambridge, MA, U.S.A., 2001; 65-87.

2. Clemens M, Weiland T. Transient eddy current calculation with the FI-method. IEEE Transactions on Magnetics 1999; 35:1163-1165.

3. Tonti E. Algebraic topology and computational electromagnetism. 4th International Workshop on Electric and Magnetic Fields, Marseille, France, 12-15 May 1998; 284-294. 
4. Tonti E. Finite formulation of the electromagnetic field. IEEE Transactions on Magnetics 2002; 38:333-336.

5. Bossavit A. How weak is the weak solution in finite elements methods? IEEE Transactions on Magnetics 1998; 34:2429-2432.

6. Bossavit A. Generalized finite differences in computational electromagnetism. In PIER 32, Teixeira FL (ed.). EMW Publishing: Cambridge, MA, U.S.A., 2001; 45-64.

7. Bossavit A. On the geometry of electromagnetism. (1): Euclidean space. The Journal of the Japan Society of Applied Electromagnetics and Mechanics 1998; 6:17-28.

8. Bossavit A. On the geometry of electromagnetism. (2): Geometrical objects. 1998; 6:114-123.

9. Bossavit A. On the geometry of electromagnetism. (3): Integration, Stokes', Faraday's laws. The Journal of the Japan Society of Applied Electromagnetics and Mechanics 1998; 6:233-240.

10. Bossavit A. On the geometry of electromagnetism. (4): Maxwell's house. The Journal of the Japan Society of Applied Electromagnetics and Mechanics 1998; 6:318-326.

11. Trevisan F, Kettunen L. Geometric interpretation of finite dimensional eddy current formulations. International Journal for Numerical Methods in Engineering 2006; 67(13):1888-1908.

12. Codecasa L, Specogna R, Trevisan F. Symmetric positive-definite constitutive matrices for discrete eddy-current problems. IEEE Transactions on Magnetics 2007; 43(2):510-515.

13. Tonti E. On the geometrical structure of electromagnetism. In Gravitation, Electromagnetism and Geometrical Structures for the 80th birthday of Lichnerowicz A, Ferrarese G (ed.). Pitagora Editrice: Bologna, Italy, 1995; 281-308.

14. Bossavit A, Kettunen L. Yee-like schemes on staggered cellular grids: a synthesis between FIT and FEM approaches. IEEE Transactions on Magnetics 2000; 36(4):861-867.

15. Codecasa L, Trevisan F. Piecewise uniform bases and energetic approach for discrete constitutive matrices in electromagnetic problems. International Journal for Numerical Methods in Engineering 2006; 65(4):548-565.

16. Albanase R, Rubinacci G. Integral formulation for $3 \mathrm{D}$ eddy-current computation using edge elements. IEE Proceedings 1988; 135:457-462.

17. Kettunen L, Forsman K, Levine D, Gropp W. Volume integral equations in non-linear 3-D magnetostatics. International Journal for Numerical Methods in Engineering 1995; 38:2655-2675.

18. Tarhasaari T, Kettunen L, Geuzaine C. Discretization of sources of integral operators. IEEE Transactions on Magnetics 2000; 36(4):659-662.

19. Albanase R, Rubinacci G. Treatments of multiply connected regions in two-component electric vector potential formulations. IEEE Transactions on Magnetics 1990; 26:650-653.

20. Kettunen L, Forsman K, Bossavit A. Formulation of the eddy current problem in multiply connected regions in terms of h. International Journal for Numerical Methods in Engineering 1998; 41(5):935-954.

21. Gross PW, Kotiuga PR. Electromagnetic theory and computation. Mathematical Sciences Research Institute Publications, vol. 48. Cambridge University Press: Cambridge, 2004.

22. Specogna R, Saku S, Trevisan F. Geometric T- $\Omega$ approach to solve eddy-currents coupled to electric circuits. International Journal for Numerical Methods in Engineering 2008; 74(1):101-115.

23. Bossavit A. Computational Electromagnetism. Academic Press: New York, 1998.

24. Stratton JA. Electromagnetic Theory. McGraw Hill: New York, 1941. 